\title{
SHORT WAVE TREATMENT METHODS
}

\section{EXTRACTS FROM A LECTURE-DEMONSTRATION GIVEN BY}

\section{Miss E. M. MEREDITH, M.C.S.P., (Teacher's Certificates)}

Member of the Teaching Staff of the Pretoria School of Physiotherapy, to the Members of the Northern Transvaal Branch, of the South African Society of Physiotherapy, in November, 1959.

Diagrams by: Miss J. WOOD (Pretoria Diploma).

\section{SHORT WAVE DIATHERMY FOR BOTH ARMS AND CERVICAL SPINE AT SAME TIME.}

A VERY satisfactory treatment can be given by using are possible.

The patient, wearing a vest only above the waist, is wrapped in a blanket below the arms, leaving arms and shoulders free, and is then seated on a chair, with each arm fully supported by pillows on a small table or arm bath stand.

Two pieces of thin blanket, approximately $48 \times 36$ inches each, are needed for spacing, and are applied (with the operator standing behind the patient) as follows:-

The top corner of short end of one is placed from the back over, say $\mathbf{R}$. Clavicle, mid way between acromion and neck, the length of the blanket lying along the L. arm. This arm is gently lifted and supported while the lower corner of the same short end is brought under the L. Axilla and up over the L. Clavicle from in front.

Next the lower (long) side of the blanket is wrapped up over part and top of whole length of the arm (see Diagram A) and then the upper long side over this first layer downwards and under, so that the whole arm has two layers of covering.

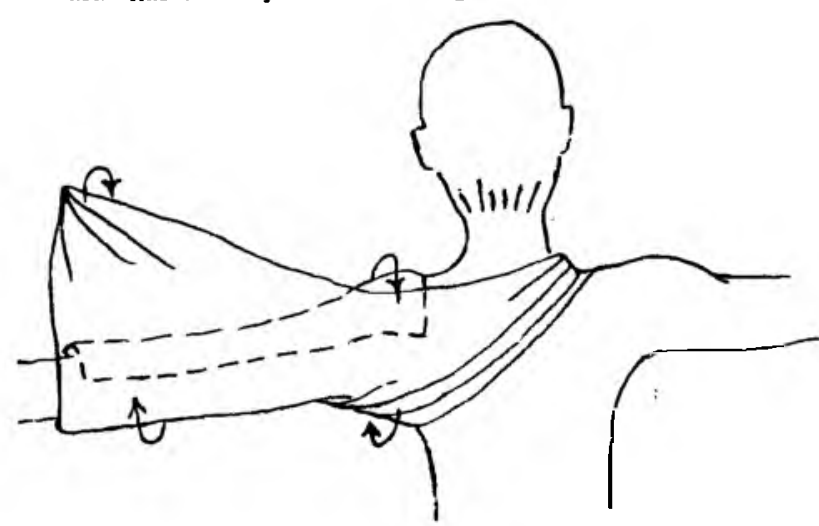

The other blanket is applied in the same way over the $\mathbf{R}$. arm, so that there are now two layers over back of neck also.

If arms are to be treated to approximately the same extent, but $L$. shoulder is more painful than $R$. shoulder, the middle of the cable is found and placed at back of neck, and one end wound spirally round the $\mathrm{L}$. arm, the first turn coming over top of shoulder from behind, and the coil extending as far as required.

The arm, of course, must be fully supported the whole time this is being done, and be moved as little as possible.

The opposite half of the cable is wound in the same direction as the first, round the $\mathrm{R}$. arm, i.e. as the first turn was brought above the L. Shoulder that of the second half must come below R. Axilla, so that whole coil is wound in anti-clockwise direction, very careful checking is needed before current is turned on, to ensure that sufficient blanket is in the axilla so that no bare turn lies against the chest wall, that coils are evenly spaced, and that the ends of the cable between patjent and apparatus are of equal length. One advantage of this arrangement is that all of both arms within the coil is heated, and also across back of shoulders and base of neck.

Another advantage is that almost any position of arms is satisfactory, i.e. any degree of abduction and/or flexion of shoulder that is comfortable for patient. The only point to watch is that if the arm is flexed that piece of cable to apparatus does not come into contact with turns round the arm.

Modifications.

If more heat is required in the neck, the middle of the coil can be arranged in a vertical loop or zig-zag with a loop reaching as high as is needed. This can be held in: place by a short bandage being tied to the middle of the loop and passed round the patient's forehead, while the distance between the loops is maintained by plastic spacers. (See Diagram B).

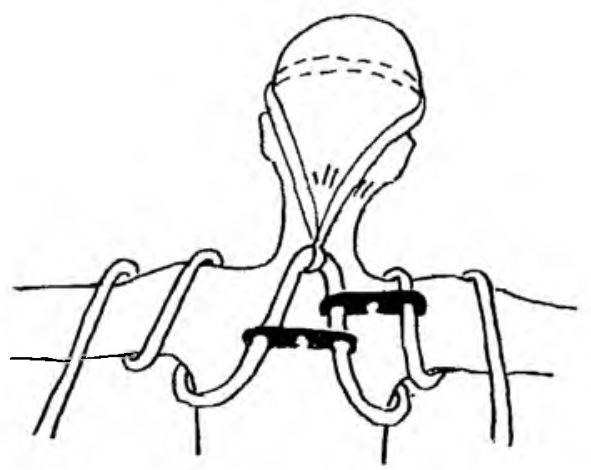

It is also very simple to vary the extent of either arm being treated, e.g. one may be treated from fingers to shoulder and the other with the shoulder only.

If $\mathbf{R}$. shoulder is more affected than $\mathbf{L}$. shoulder coil is wound in opposite direction, clockwise, being first placed over R. shoulder. If both are equally painful and need same intensity, the top coil on the second arm can be brought to top of shoulder, and held by spacer between it and that part of the cable across the back (see Diagram B).

With this method cause-cervical lesion-and symptom -pain in arms - can be treated at the same time, and this usually has a very beneficial effect on patient, relief of pain being produced more quickly than if cervical spine only is treated.

\section{SHORT WAVE TO HIP JOINT.}

One method that is valuable to all cases when hip joint is to be treated, and is the method par excellence if there is flexion deformity, is to give a co-axial current by using one large fiexible electrode, and a short inductothermy cable as a condenser elect rode.

The patient is fully supported in half lying on a plinth, and can wear a vest and cotton knickers. The trunk and thighs should be as nearly at right angle as the comfort of the patient will allow.

The flexible pad with 2 or 3 felt spacers is placed behind the affected hip, well down. (An equal thickness of pillow or toweling should be placed behind the opposite hip so that pressure is even).

The cable is connected to the apparatus by one end only, the other end being heavily insulated by a rubber bandage or other equivalent. It is then wound about 3 times round 
the thigh, the free end being nearer the hip than the knee.

The effect of this is to produce a virtual condenser electrode placed at cross section of the limb, i.e. parallel with one behind the hip with the joint on the axial line between them; and a longitudinal or co-axjal current will pass from one to the other. There is little or no magnetic effect so that the turns of the coil can be close to one another.

The opposite thigh must of course be sufficiently separated from the coil by air, a pillow or toweling.

As field concentration on skin is very much diminished and pathway is wide more current can be given, and Patients say they can feel the warmth deep, really " $i n$ " the hip, and find it most comfortable.

An apparatus with an adequate output and variable condenser is essential as the electrode-patient capacity is large. If, however, tuning is difficult because variable condenser is inadequate, smaller pad electrode and/or wider spacing of the turns of the coil will probably diminish the capacity enough to make tuning possible.

The apparatus is placed at side of patient so that there is no trouble in keeping leads parallel and uncrossed, but generally speaking the length of cable between patient and apparatus should be shorter than the lead of the pad, but this will depend upon the position of voltage antinode in the cable.

If there are different sockets for condenser and cable electrodes those for the condensers are used as a rule.

\section{CASE HISTORY NOTES}

(Taken from the Patient's own report.)

Mr. M. developed pain in the right eye in 1945, which became severe and gradually spread to the mouth, tongue, soft plate and right side of the head, causing the eye to water, tongue to swell, and producing a heavy discharge from the right nostril; also perspiration on the right side of forehead and temple during an attack of pain.

These attacks of pain became very frequent and of varying lengths of duration. They were brought on by activities of the arms and neck, such as lifting, golf, lying in a concave bed, driving a motor car and through emotional upsets.

Various diagnoses were made and the appropriate treatments given, but all were of no avail. Drugs such as aspirin and others much more potent afforded no relief. Later following the diagnosis of Tic doloreux alcoholic injections into the trigeminal nerve, produced negative results, as did a sympathectomy and anti-histamine treatments for migraine.

Eventually the patient was referred to Mr. M. D. Oliver, M.C.S.P. of Cape Town with diagnosis "unknown", except that a note was made of an old injury to the neck and back some twenty years previous while playing rugby.

Treatment by Physiotherapist:

Sustained Traction by means of the Rosslyn traction couch. A pull of $20 / 25$ lbs. gave slight relief but a pull of 45 lbs. which was felt right down the spine produced considerable improvement and no discomfort was experienced.

Postural Exercises.

The patient had for years stood in a stooped position because this seemed to ease the pain. Corrective postural exercises were taught and these gave tremendous relief.

Shoulder and Neck Mobilising Exercises.

The range of neck and shoulder movement was very limited and by excessive movement an attack of pain could be produced. The patient was taught various self mobilising exercises to increase the range of neck and shoulders and these were strictly adhered to.

Result.

During the last 6 months there has been no recurrence of the pain and discomfort experienced from 1945 to the end of 1958.

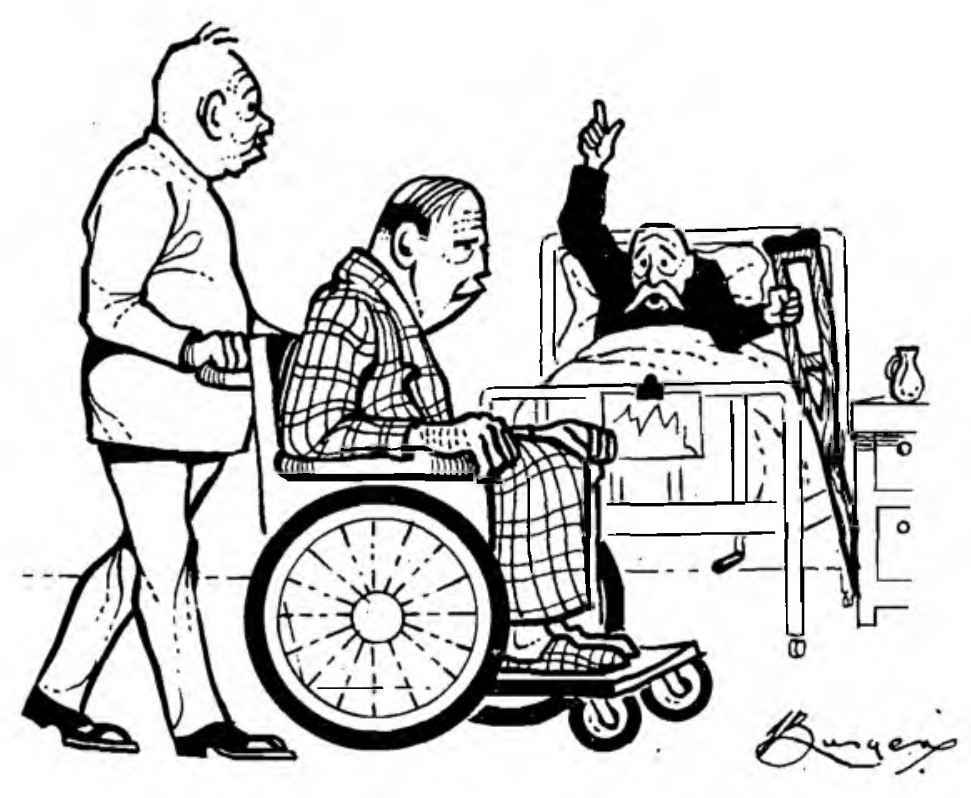

valuable assistance

during convalescence

with the aid of a

wheel chair or

crutches hired from

Clinical Emergencies,

Johannesburg, who also

have 'walking machines',

infra red lamps, etc.,

for hire. 